\title{
Interference effects in soliton scattering by impurities
}

\author{
Yuri S Kivshar $\dagger \neq \|$, Angel Sánchez $\dagger$, Oksana Chubykaloł \$; \\ Arnold M Kosevich $\ddagger$ and Luis Vázquez† \\ † Departamento de Física Teórica I, Universidad Complutense, Ciudad Universitaria, \\ E-28040 Madrid, Spain \\ ¥ Institute for Low Temperature Physics and Engineering, 47 Lenin Avenue, Kharkov \\ 310164, Ukraine \\ 8 Kharkov State University, Dzerzhinskaya Square 4, Kharkov 310007, Ukraine
}

Received 27 May 1992

\begin{abstract}
We present a comprehensive study of the effects of inhomogeneities on nonlinear excitation dynamics in different one-dimensional soliton-bearing systems. We theoretically analyse soliton scattering by one and two point-like impurities in the framework of the perturbed sine-Gordon, nonlinear Schrödinger and Konteweg-de Vries equations, and predict that interference effects arise in the form of an oscillatory dependence of the soliton reflection coefficient on the distance between impurities. The main factor responsible for this behaviour is the spectral density of the soliton radiation: the values of that distance which are integer multiples of the main wavelength radiated by the soliton upon collision with the inhomogeneities give rise to resonant scattering, with very little reflection. Our approximate analytical predictions explain previous numerical results concerning nonlinear lattices, and are further supported by new simulations that we have performed on the sine-Gordon system.
\end{abstract}

\section{Introduction}

The comprehension of the interplay between disorder and nonlinearity is of fundamental importance in many physical contexts. Their combination gives rise to a number of unsolved mathematical and physical problems, which are currently receiving much attention [1]. Most of these problems belong to condensed matter physics, where nonlinear, soliton-bearing models are often used to approximately describe actual systems, and disorder is later taken into account to make models more realistic (see, for instance, [2] and [3] for reviews). One of the most interesting and natural questions thus arising is that of the mutual influence between the effects related to Anderson localization and the possibility of soliton propagation. As a first step to understand this relationship, it is very important to have a well-established picture of soliton scattering in systems with few impurities, because localization generally originates from multiple interference between backscattered waves.

Solitons, the well known self-localized waves which describe elementary nonlinear excitations in many physical systems, can be seen as particle-like objects for many purposes [4]. This is so because the evolution equations, derived through some analytical approach, that govern their dynamics under the influence of perturbations, are

|| Present address: Institut für Theoretische Physik I, Heinrich-Heine-Universität Düsseldorf, D-4000 Düsseldorf 1, Federal Republic of Germany. 
commonly quite similar to those of particles under external forces. However, as solitons are solutions of nonlinear wave equations, they possess also wave properties: for instance, solitons emit small amplitude (quasi-linear) radiation upon collision with a single inhomogeneity $[2,3]$. This radiation could then originate interference phenomena when several scatterers are present in the system, which would constitute the most remarkable manifestation of the corpuscular-wave character of solitons.

On the other hand, aside from the fact that they all share a few, very general features, solitons can be classified in three different types [5]: dynamical solitons, which are sustained by means of dynamical conservation laws, like Korteweg-de Vries (Kdv) ones; topological solitons, which connect parts of the systems in different states that have the same energy, as in the nonlinear Klein-Gordon models, and envelope solitons, which consist of a carrier wave with a localized envelope, as in the nonlinear Schrödinger (NLS) equation. The distinction between solitons belonging to one kind or another arises from many factors, and it is quite likely that their scattering properties will also not be the same. This is the reason why each one of these classes deserves a separate study on its own, as addressed in this paper. As our main aim is related to interference effects, we will deal with the problem of soliton scattering by two point-like impurities, the simplest model capable of giving rise to these effects.

The paper is organized as follows. In section 2 we review, for completeness, the main general results of soliton perturbation theory, which we will use to analyse the radiation induced effects in inhomogeneous systems. Section 3 is devoted to the study of nonlinear chains, in which both dynamical solitons and envelope solitons are allowed to propagate, being described by effective KdV and NLS equations, respectively. We will show that the former does not show resonant scattering for any distance between impurities, while the latter does have an oscillatory dependence of the soliton reflection coefficient on the distance between impurities. By means of these results, we will be able to explain the outcome of previous numerical work on nonlinear lattices [6]. In section 4 we prove a similar behaviour for the remaining kind of solitons, topological ones, taking the sine-Gordon system as an example. We make a detailed comparison of our predictions with numerical simulations specifically designed and performed for this purpose on the sine-Gordon and $\phi^{4}$ models, and finish with some comments on the physical relevance of these calculations. Section 5 concludes the paper.

\section{Perturbation induced dynamics of solitons}

\subsection{Adiabatic dynamics of solitons}

Soliton dynamics under the action of external inhomogeneous perturbations has been studied in a number of papers (e.g. see $[2,3]$ and references therein). In particular, starting from the famous paper by Fogel et al [4], static inhomogeneities which describe several local defects have been considered as perturbations in all nonlinear models. If these inhomogeneities are not too strong, or do not vary too fast, their effect on soliton dynamics can be estimated by means of perturbative procedures. More specifically, these procedures apply in two cases, namely, if the characteristic size of the impurities is either much larger or much smaller than the soliton size; then, the ratio of both lengths can be used as a small parameter to develop the corresponding approximate description of soliton dynamics through perturbation theory. In this framework, the crudest approach is the zeroth order one: to suppose that the influence 
of the inhomogeneities on the soliton gives rise only to change in their parameters, like centre position, velocity, etc. This amounts to considering them as particle-like objects as we stated above, and it is usually named the adiabatic approach.

As an illustration, let us briefly consider the motion of a sine-Gordon ( $\mathrm{sG}$ ) soliton, also referred to as a kink, in an external field, described by the equation

$$
u_{t z}-u_{x x}+\sin u=\varepsilon f(x) \sin u
$$

where the subscripts $t$ and $x$ stand for partial derivatives with respect to the corresponding variables. When $\varepsilon=0$, the kink solution of the ${ }_{s} G$ equation is given by

$$
u_{k}(x, t) \equiv 4 \tan ^{-1} \exp \left[\sigma \frac{x-\zeta}{\sqrt{1-V^{2}}}\right]
$$

where $\zeta=V t+\zeta_{0}$ is the kink centre coordinate, $V$ is its velocity and $\sigma= \pm 1$ ( $\sigma=+1$ corresponds to a kink, $\sigma=-1$ to an antikink). Besides, the unperturbed sG system has an infinite number of quantities that are conserved in the evolution, among which there is the momentum,

$$
P \equiv-\int_{-\infty}^{\infty} \mathrm{d} x u_{t} u_{x} .
$$

For the kink, equation (2.2), equation (2.3) takes the form of the well known relativistic expression $P=8 V / \sqrt{1-V^{2}}$. In the presence of perturbations, the momentum is no longer conserved; using (2.1) it is possible to show that it varies according to

$$
\frac{\mathrm{d} P}{\mathrm{~d} t}=\varepsilon \int_{-\infty}^{\infty} \mathrm{d} x f(x)(\cos u)_{x}
$$

which is valid provided the boundary condition $u \rightarrow 0(\bmod 2 \pi)$ at $x \rightarrow \pm \infty$ holds. The adiabatic approach is now defined by the assumption that, for $\varepsilon$ small enough, the kink shape will not be affected and its coordinate $\zeta$ and its velocity $V$ will become slowly changing functions of time. Within this hypothesis it can be then shown that, in the non-relativistic limit $V^{2} \ll 1$, the kink centre obeys the following evolution law:

$$
8 \frac{\mathrm{d}^{2} \zeta}{\mathrm{d} t^{2}}=4 \varepsilon \int_{-\infty}^{\infty} \mathrm{d} x \frac{f(x) \sinh (x-\zeta)}{\cosh ^{3}(x-\zeta)} \equiv-\frac{\mathrm{d}}{\mathrm{d} \zeta} U(\zeta)
$$

where

$$
U(\zeta) \equiv-2 \varepsilon \int_{-\infty}^{\infty} \mathrm{d} x \frac{f(x)}{\cosh ^{2}(x-\zeta)}
$$

and we have used the approximate expression $P \approx 8 \mathrm{~d} \zeta / \mathrm{d} t$, valid for small velocities. Thus, in the framework of the adiabatic approach, the motion of the sG kink can be thought of as that of a particle with mass 8 in the external potential $U(\zeta)$ defined by (2.6). The same can be shown for relativistic kinks [7].

The two aforementioned cases arise naturally from equations (2.5) and (2.6). If $f(x)$ changes rapidly over distances of the order of the kink length, then $\varepsilon$ has to be small for our approximation to hold. For example, in the case $f(x)=\delta(x)$, we have [8]

$$
U(\zeta)=-2 \varepsilon \operatorname{sech}^{2}(\zeta)
$$


On the other hand, if $f(x)$ changes slowly, i.e. its characteristic length (say $L$ ) is much larger than the kink length, it is not necessary that $\varepsilon$ is small, because all the parameters of the perturbation theory are of the order of $L^{-1}$, and we are left with

$$
U(\zeta)=-2 \varepsilon \int_{-\infty}^{\infty} \mathrm{d} x f\left(\frac{x+\zeta}{L}\right) \operatorname{sech}^{2} x \approx 4 \varepsilon f\left(\frac{\zeta}{L}\right) .
$$

Up to now, we have seen that soliton dynamics in the presence of inhomogeneities is similar to that of particles in external fields. However, solitons are solutions of nonlinear evolution equations, which describe systems with an infinite number of degrees of freedom. Hence, the reduction of such a complicated problem to one with one or two degrees of freedom is an approximate, oversimplified procedure. The actual soliton dynamics is accompanied by emission of linear waves both forwards and backwards, and this emission cannot be accounted for in the adiabatic approach. This is the topic we deal with in the next subsection.

\subsection{Perturbation-induced soliton emission}

The most powerful method to analyse wave properties of solitons, i.e. those related to their emission induced by any kind of perturbation, is the one based in the inverse scattering transform (IST) [9], which, in turn, can be only used for systems close to completely integrable ones. In recent years, perturbative techniques of this type have been developed for almost all of the quasi-integrable systems (see [2] for a detailed account of these techniques). The general idea of the procedure is the following. For the unperturbed system, the IST provides a transformation from the nonlinear evolution equation to a linear integral problem. This transformation is in some sense similar to an action-angle change of variabies: the evolution of the wavefield can be written in terms of the eigenvalues of a spectral problem, which remain constant while the field evolves in time, and of the corresponding eigenfunctions, which have a trivial evolution. For instance, the eigenfunctions associated with the radiation, usually represented by $b(\lambda, t)$ (the so-called Jost coefficients, see, e.g. [9]), $\lambda$ being the eigenvalue, follow the equation

$$
\frac{\partial b(\lambda, t)}{\partial t}=\mathrm{i} \Omega(\lambda) b(\lambda, t)
$$

where $\Omega(\lambda)$ is the radiation frequency. Its precise relationship to $\lambda$ depends on the specific equation we are considering.

If we now add to the unperturbed equation a new term, say $\varepsilon R(u)$, the time evolution of these Jost coefficients change, and it can be shown to be given, up to first-order terms, by

$$
\frac{\partial b(\lambda, t)}{\partial t}=\mathrm{i} \Omega(\lambda) b(\lambda, t)+\varepsilon F(\lambda, t)
$$

where

$$
F(\lambda, t) \equiv \int_{-\infty}^{\infty} \mathrm{d} x \frac{\delta b(\lambda, t)}{\delta u(x, t)} R(u) .
$$

Assuming that at $t \rightarrow-\infty$ the wavefield $u$ had the shape of an exact solution of the unperturbed problem, which amounts to saying that there was no radiation at all, we 
can put $b(\lambda,-\infty)=0$. Subsequently, the solution of $(2.10)$ at $+\infty$ can be obtained as

$$
b(\lambda, t) \stackrel{t \rightarrow \infty}{\longrightarrow} \varepsilon \mathrm{e}^{\mathrm{i} \Omega(\lambda, t) t} \int_{-\infty}^{\infty} \mathrm{d} t^{\prime} F\left(\lambda, t^{\prime}\right) \mathrm{e}^{-\mathrm{i} \Omega(\lambda, t) t} \equiv \mathrm{e}^{\mathrm{i} \Omega(\lambda, t) t} B(\lambda) .
$$

Finally, the suitable physical quantity to describe the radiation emission, the emitted spectral energy density $\mathscr{E}(\lambda)$, can be estimated when the radiated amplitude is not too large $\left[|b(\lambda, t)|^{2} \ll 1\right]$ through

$$
\mathscr{E}(\lambda) \approx \frac{1}{\pi} g(\lambda)|b(\lambda, t)|^{2}
$$

where $g(\lambda)$ again depends on the precise equation we are studying, and can be computed by means of IST. Thus, in this approximation the total energy emitted by a soliton during its interaction with the perturbation is

$$
\mathscr{E}(\lambda, t=+\infty)=\frac{1}{\pi} g(\lambda)|B(\lambda, t)|^{2}
$$

and

$$
E_{\mathrm{rad}}=\int_{\lambda_{\min }}^{\lambda_{\max }} \mathrm{d} \lambda \mathscr{E}(\lambda, t=+\infty)
$$

this last one being the fundamental expression that we will need in what follows.

\section{Soliton-impurity interaction in nonlinear chains}

Now, let us apply the formalism that we have briefly reviewed to specific problems. We will begin studying a nonlinear monoatomic chain, with nearest-neighbour interactions, whose Hamiltonian is

$$
H=\sum_{n}\left[\frac{1}{2} m_{n}\left(\frac{\mathrm{d} y_{n}}{\mathrm{~d} t}\right)^{2}+U\left(y_{n}-y_{n-1}\right)\right] .
$$

Here, $y_{n}(t)$ stands for the longitudinal displacement of the $n$th atom from its equilibrium position, and $m_{n}=\gamma m$, with $\gamma \neq 1$ only for certain sites, which represent the impurities. With respect to the interaction potential, we can choose it in a rather general form, with terms up to fourth order, as

$$
U(r)=\frac{G r^{2}}{2}+\frac{A r^{3}}{3}+\frac{B r^{4}}{4}
$$

This will be a good approximation to any potential for not too large displacements. From the Hamiltonian (3.1), the equations of motion can be written in the form

$$
\begin{gathered}
m_{n} \frac{\mathrm{d}^{2} y_{n}}{\mathrm{~d} t^{2}}=G\left(y_{n+1}-2 y_{n}+y_{n-1}\right)+A\left(y_{n+1}-y_{n}\right)^{2}-A\left(y_{n}-y_{n-1}\right)^{2} \\
+B\left(y_{n+1}-y_{n}\right)^{3}-B\left(y_{n}-y_{n-1}\right)^{3} .
\end{gathered}
$$

It is possible to obtain approximate solutions for the set of equations (3.3) in the well known continuum limit [10]. In such a limit, one can derive either the Boussinesq (Bq) equation or the NLS equation, depending on the type of excitations considered, as we will show in the next two subsections. 


\subsection{Dynamical kinks}

3.1.1. Equation of motion and kink solution. Looking for kink-like solutions and using an improved quasi-continuum approximation [11], equation (3.3) can be cast in the form of a generalized Boussinesq equation $(\mathrm{GBq})$ (for the corresponding derivation in the perfectly homogeneous case, see $[6,10])$, given by

$$
u_{t t}-c^{2} u_{x x}-p\left(u_{x}^{2}\right)_{x}-q\left(u_{x}^{3}\right)_{x}-h u_{x x x x}=a(1-\gamma) f(x) u_{t t}
$$

where $\boldsymbol{a}$ is the lattice spacing, $\boldsymbol{u} \equiv y_{x}$ and

$$
c^{2} \equiv \frac{G a^{2}}{m} \quad p \equiv \frac{A a^{3}}{m} \quad q \equiv \frac{B a^{4}}{m} \quad h \equiv \frac{G a^{4}}{12 m}
$$

are constants. On the other hand, the function $f(x)$ may describe several inhomogeneities; for example, we will be considering below the two-impurity problem, $f(x)=\varepsilon[\delta(x)+\delta(x-D)]$.

In the absence of any perturbation and when $q=0$ (the standard Bq equation), equation (3.4) has a kink-like solution of the form

$$
u(x, t)=A_{m} \tanh \left(\frac{x-V t}{L}\right)
$$

where

$$
A_{m} \equiv \frac{2 \operatorname{sgn}(h)}{p} \sqrt{h\left(V^{2}-c^{2}\right)} \quad L \equiv 2 \sqrt{\frac{h}{V^{2}-c^{2}}}
$$

$V$ being the kink velocity; on the other hand, the kink energy is given by

$$
E_{\mathrm{k}}=\frac{4}{15} \frac{m A_{m}^{2}}{a L}\left(4 V^{2}+c^{2}\right)
$$

This is the solution whose scattering properties we want to study. However, as it stands, the problem is still not suitable for our perturbative approach, because the unperturbed GBq equation (3.4) with $f(x)=0$ is not integrable. To be able to obtain the analytical results we are interested in, it has to be transformed once more. Let us introduce the new variables

$$
\zeta \equiv \varepsilon(x-c t) \quad \tau=\varepsilon^{3} t
$$

and look for solutions of the form

$$
u(x, t)=\varepsilon u_{1}(x, t)+\varepsilon^{3} u_{3}(x, t)+\ldots
$$

Substitution of (3.9) and (3.10) into (3.4) with $q=0$ yields a perturbed $\mathrm{KdV}$ equation for the function $w \equiv\left(u_{1}\right)_{\zeta}$ :

$$
2 c w_{\tau}+p\left(w^{2}\right)_{\zeta}+h w_{\zeta \zeta \zeta}=-\left(\frac{\tilde{y}}{\varepsilon^{2}}\right) f\left(\zeta+\frac{c \tau}{\varepsilon^{2}}\right)\left(c^{2} w_{\zeta}-2 \varepsilon^{2} w_{\tau}\right)
$$

with $\tilde{\gamma} \equiv a(1-\gamma)$.

If we finally introduce the variables

$$
\tilde{\tau} \equiv \frac{\tau}{2 c \sqrt{h}} \quad \tilde{\zeta} \equiv \frac{\zeta}{\sqrt{h}} \quad \tilde{w}=-\frac{p w}{2}
$$


we obtain the usual KdV equation when $\tilde{\gamma}=0$. Thus, we can now profit from the perturbation theory for the KdV soliton to study the effects of the additional term

$\varepsilon R(u)=-\left(\frac{\tilde{\gamma}}{h \varepsilon^{2}}\right) f\left(\zeta+\frac{2 c^{2} \tilde{\tau}}{\varepsilon^{2}}\right)\left(c^{2} \tilde{w}_{\tilde{\zeta}}-\varepsilon^{2} \tilde{w}_{\tilde{\tau}}\right) \approx\left(\frac{\tilde{\gamma}}{2 h}\right) f\left(\tilde{\tau}+\frac{\varepsilon^{2}}{2 c^{2}} \tilde{\zeta}\right) \tilde{w}_{\tilde{\zeta}}$

on the KdV soliton

$$
w(\zeta, t)=\frac{4 \kappa^{2}}{p \cosh \left[\kappa / \sqrt{h}\left(\zeta-\left(4 \kappa^{2} / 2 c\right) \tau\right)\right]} .
$$

A direct comparison of (3.14) to the expansion of (3.6) and (3.7) for small values of the parameter $\Delta \equiv \sqrt{V^{2}-c^{2}}$ leads to the relation

$$
\varepsilon \kappa=\frac{1}{2} \sqrt{V^{2}-c^{2}}=\frac{1}{2} \Delta
$$

and in the same approximation the kink energy takes the form

$$
E_{\mathrm{k}} \approx \frac{4 m \sqrt{h}}{3 a p^{2}} c^{2} \Delta^{3} \text {. }
$$

3.1.2. Kink radiative effects. We can now compute the reflection coefficient for the dynamical kink. To this end, we will rewrite the energy (3.1) as an integral of the $\mathrm{KdV}$ equation by means of the new variables given in (3.9) and (3.12) and of the continuum approximation, obtaining

$$
E=\frac{2 m c^{2} \sqrt{h}}{p^{2} a} \varepsilon \int_{-\infty}^{\infty} \mathrm{d} \tilde{\zeta} \tilde{w}
$$

The spectral density $\mathscr{E}(\lambda)$ of the energy radiated by the kink can be calculated with the help of the formalism of section 2 and using the formulae from the review paper [2]. For a single impurity, i.e. $f(x)=\delta(x)$, we arrive at

$$
|B(\lambda,+\infty)|^{2}=\frac{2^{6}}{9} \pi^{2}\left(\frac{\tilde{\gamma} \kappa^{2} \varepsilon^{2}}{4 h c^{2}}\right)^{2} \frac{b^{2}\left(1+b^{2}\right)^{2}}{\sinh ^{2}(\pi b)} \quad b=\frac{\lambda}{\kappa}
$$

and hence the spectral density is given by

$$
\mathscr{E}(\lambda)=\frac{8 \lambda^{2}}{\pi}|B(\lambda)|^{2}=\frac{2 \pi}{9}\left(\frac{\tilde{\gamma}}{h c^{2}}\right) \Delta^{4} \frac{b^{4}\left(1+b^{2}\right)^{2}}{\sinh ^{2}(\pi b)} .
$$

The total energy reflected by the impurity (recall that KdV solitons can emit linear waves only backwards, i.e. in the direction opposite to its own propagation) has the form

$$
\begin{aligned}
E_{\mathrm{em}} & =\frac{2 m c^{2} \sqrt{h}}{p^{2} a} \varepsilon \int_{0}^{\infty} \mathrm{d} \lambda \mathscr{E}(\lambda)=\int_{0}^{\infty} \mathrm{d} b E_{\mathrm{em}}(b) \\
& =\frac{2 m c^{2} \sqrt{h}}{9 p^{2} a} \Delta^{5}\left(\frac{\tilde{\gamma}}{h c^{2}}\right)^{2} \int_{0}^{\infty} \mathrm{d} b \frac{b^{4}\left(1+b^{2}\right)^{2}}{\sinh ^{2}(\pi b)}
\end{aligned}
$$

which leads to the following reflection coefficient:

$$
R_{1}=\frac{E_{\mathrm{em}}}{E_{\mathrm{k}}}=\frac{\pi}{6} \Delta^{2}\left(\frac{\tilde{\gamma}}{h c^{2}}\right) \int_{0}^{\infty} \mathrm{d} b \frac{b^{4}\left(1+b^{2}\right)^{2}}{\sinh ^{2}(\pi b)}
$$

Thus, our procedure yields an $R \sim E_{k}^{2 / 3}$ as can be seen from (3.21), in good agreement with previous results of Yoshida and Sakuma [12]. Furthermore, this prediction also 
agrees very well with the numerical simulations of Qiming et al [6]. In that work a nonlinear chain was studied, but with an interparticle potential of the form $V(r)=$ $P(\exp (-\beta r)-1)^{2}$, i.e. the Morse potential, which can be approximated for small $r$ by a cubic potential like the one we have considered. They numerically obtained the quantity $S \equiv T^{-1}-1$; when $R \gg 1$ the value $S$ coincides very accurately with $R$. So, their results can be compared directly with ours, and it can be seen [6] that the numerical dependences are again $R \sim E_{\mathrm{k}}^{2 / 3}$ and $R \sim(\gamma-1)^{2}$, qualitatively the same as the ones we have computed.

The spectral density of the emitted energy, given by (3.19), $E_{\text {em }}(b) \sim \mathscr{E}(\lambda)$, increases with $b$ for small $b$, but if $b \gg 1$ it becomes exponentially small, $E_{\text {em }}(b) \sim b^{8} \exp (-2 \pi b)$ (see figure 1). Hence, it has a maximum, which is of the order of unity, at $b \sim 1$ (i.e. $\lambda \sim \kappa)$, with a rather large width. Thus, such a wide spectral density should not give rise to any particular length scale producing interference effects during KdV-soliton scattering by two impurities, which is our main interest. To prove this, let us calculate the corresponding reflection coefficient: substituting now $f(x)=\delta(x)+\delta(x-D)$, it is possible to arrive at

$$
R=\frac{2 \pi}{3}\left(\frac{\tilde{\gamma}}{h c^{2}}\right) \Delta^{2} \int_{0}^{\infty} \mathrm{d} b \frac{b^{4}\left(1+b^{2}\right)^{2}}{\sinh ^{2}(\pi b)} \cos ^{2}\left[2 A b\left(1+b^{2}\right)\right]
$$

where

$$
A \equiv \frac{D \Delta^{3}}{c^{2} \sqrt{h}} \equiv \frac{D}{c^{3} \sqrt{h}}\left(V^{2}-c^{2}\right)^{3 / 2} .
$$

For $A \gg 1$ (large distance between impurities, or large $V$ ) the asymptotic dependence turns out to be

$$
R \simeq 2 R_{1}-\frac{(2 \pi)^{3 / 2}\left(\frac{\tilde{\gamma}}{h c^{2}}\right)^{2} \Delta^{2}}{3^{21 / 4} \sin ^{2}(\pi / \sqrt{3})} \exp \left(-\frac{4 A}{3 \sqrt{3}}\right)
$$

with $R_{1}$ being defined by (3.21); as should happen, this expression implies that for separated impurities, the total reflection coefficient is simply the sum of the individual ones. At the other limit, $A \ll 1$ (nearby impurities or low velocities), we have

$$
R \simeq 4 R_{1}
$$

which is again reasonable, because if $D \rightarrow 0$ the intensity of the composite impurity becomes $2 \tilde{\gamma}$ instead of $\tilde{\gamma}$.

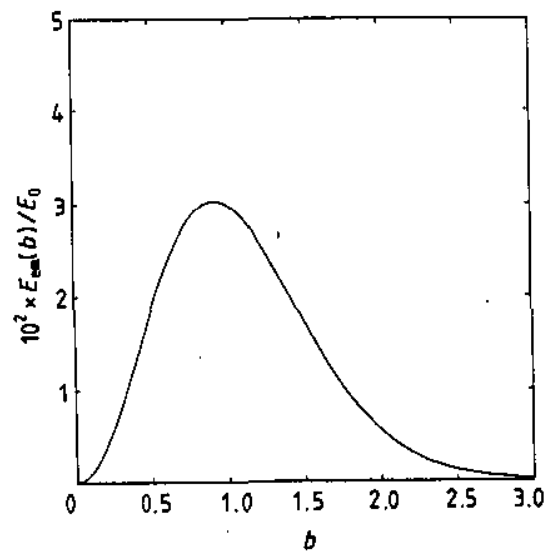

Figure 1. Spectral density emitted by a dynamical kink (a KdV soliton) in a monoatomic chain with a single inhomogeneity. The plotted curve is $E_{\text {em }}(b) / E_{0}$, with $E_{0}=2 \pi m c^{2} \sqrt{h} \Delta^{5} \tilde{\gamma}^{2} / 9 a\left(p h c^{2}\right)^{2}$, and $b=\lambda / \kappa$ (see equations (3.19) and (3.20)). 
The whole dependence of the ratio $R / 2 R_{1}$ is depicted in figure 2 . It is clearly appreciated from that plot the absence of any oscillation, which would indicate the existence of interference effects. These effects are inhibited due to the fact that there is no predominant wavenumber in the radiation emitted by the kink; instead, a wide spectrum arises. It is important to note that in the paper by Qiming et al [6], the problem for two impurities was also addressed. Those authors found a decreasing of the transmission coefficient, equivalently, an increasing of the reflection coefficient, as appears from figure 7 in [6]. This behaviour is once more qualitatively the same as that we have obtained, except for the fact that the numerical curve does not exhibit the first part of our curve, only the part after the maximum (in the transmission coefficient). The reason for this discrepancy is that the position of the minimum we are predicting is of the order of the lattice spacing used in [6] and, as a consequence, it was not possible to see numerically the rapid change of the reflection coefficient for small distances.

\subsection{Envelope solitons}

3.2.1. Equation of motion and soliton solution. Let us now move to a different type of excitation, namely, envelope solitons. To this end, we look for oscillating, localized solutions of (3.3) in the form

$$
y_{n}(t)=\sum_{j} \varepsilon^{j}\left[\sum_{m} F_{j m}(n, t) \mathrm{e}^{\mathrm{i} m \theta_{n}}+\mathrm{CC}\right]
$$

(cC stands for complex conjugate) with $\theta_{n}=k a n-\omega$ and $\varepsilon$ a small scaling parameter. By means of this ansatz, we can treat the phases $\theta_{n}(t)$ exactly and use the continuum approximation only for the envelope functions $F_{j m}(n, t)$. For the quartic potential $(p=0$ in (3.4)) it can be seen [10] that only the term with $j=m=1$ contributes in the leading order, and then (3.26) reduces to

$$
y_{n}(t)=\varepsilon F_{11}(n, t) \mathrm{e}^{\mathrm{i} \theta_{n}}+\text { cc. }
$$

The amplitude $F_{11}(n, t)$ becomes $F(x=a n, t)$ in the continuum limit and verifies the following perturbed NLS equation, accurate up to order $\varepsilon^{3}$ :

$$
\mathrm{i} \tilde{F}_{\tau}+\tilde{F}_{b \zeta}+2|\tilde{F}|^{2} \tilde{F}=\frac{\tilde{\gamma}}{2}\left(\frac{\mu \omega}{V_{\mathbf{g}}} \tilde{F}-\frac{\mathrm{i} \mu}{V_{\mathrm{g}}} \tilde{F}_{\tau}+\mathrm{i} \tilde{F}_{\zeta}\right) f\left(\tau+\frac{\mu}{V_{\mathbf{g}}} \zeta\right)
$$

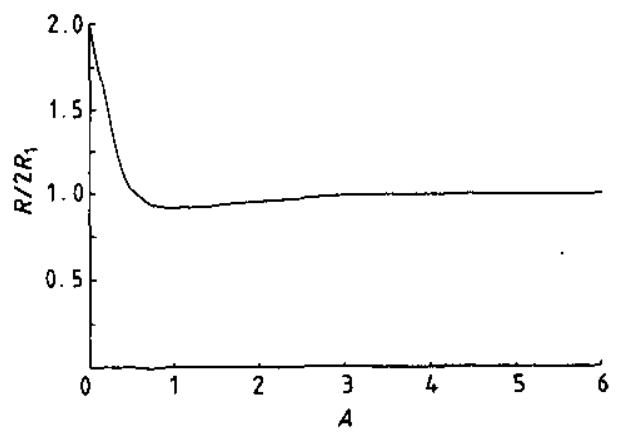

Figure 2. Ratio of soliton reflection coefficients $R / 2 R_{1}$ against the parameter $A$ defined in equation (3.23), which is proportional to the distance between impurities. 
where $f(x)$ is once more related to possible inhomogeneities, $\tilde{\gamma}$ is the same as in the preceding section, and

$$
\begin{aligned}
& \tilde{F} \equiv \sqrt{\frac{k_{0}}{2}} F \\
& k_{0} \equiv-\frac{24}{\omega \mu} \frac{B}{m} \sin ^{4}\left(\frac{k a}{2}\right) \\
& \omega^{2} \equiv 4\left(\frac{G}{m}\right) \sin ^{2}\left(\frac{k a}{2}\right) \\
& V_{\mathrm{g}} \equiv \frac{\partial \omega}{\partial k}=a \sqrt{\frac{G}{m}} \cos \left(\frac{k a}{2}\right) \\
& \mu \equiv \frac{\partial^{2} \omega}{\partial k^{2}}=-\frac{a^{2}}{2} \sqrt{\frac{G}{m}} \sin \left(\frac{k a}{2}\right)
\end{aligned}
$$

and the variables in (3.28) are

$$
\tau \equiv \mu t \quad \zeta \equiv x-V_{\mathrm{g}} t .
$$

A similar approach applies also for the case of cubic and cubic plus quartic potentials, except for the fact that it is necessary to keep consistently the zero- and second-order terms in $\varepsilon$ and some higher harmonics, even though few of them contribute (e.g. see [10]).

When $\tilde{\gamma}=0$ and $k_{0}>0$, equation (3.28) admits the well known envelope soliton solution, whose expression is

$$
\tilde{F}_{k}(\zeta, \tau) \equiv 2 \mathrm{i} \eta \frac{\exp (\mathrm{i} \xi z / \eta)+\mathrm{i} \delta}{\cosh z}
$$

where $2 \eta$ is the soliton amplitude, $-4 \xi$ its velocity, and

$$
z \equiv 2 \eta\left(x+4 \xi t-\xi_{0}\right) \quad \delta \equiv 4\left(\eta^{2}+\xi^{2}\right)+\delta_{0} .
$$

As in the previous section, we will concern ourselves with the problem of this soliton (3.35) scattering by one or two impurities.

3.2.2. Soliton radiative effects. Let us begin with the single impurity problem, corresponding to the choice $f(x)=\delta(x)$. It can be verified that only the last term from (3.28) contributes to the function $B(\lambda)$ in this case, and that, after direct but cumbersome calculations for the perturbation (for more details, see $[2,13]$ )

$$
\varepsilon R(\tilde{F})=\frac{\mathrm{i} \tilde{\gamma}}{2} \tilde{F} \delta\left(\tau+\frac{\mu}{V_{\mathrm{g}}} \zeta\right)
$$

one arrives at

$$
|B(\lambda)|^{2}=\frac{4 \tilde{\gamma}^{2} \mu^{2}}{V_{\mathrm{g}}^{2}} \pi^{2} \frac{(\lambda-\xi)^{2}+\eta^{2}}{\cosh ^{2}(\pi(\lambda-\xi) / 2 \eta)} .
$$

To recast this expression into a physical meaningful one, let us transform the chain energy using the same approximations described above: substitution of (3.26) and 
(3.34) into the chain Hamiltonian (3.1) leads, in the lowest order, to the following expression for the energy:

$$
E=\frac{m \omega^{2}}{k_{0}} \int_{-\infty}^{\infty} \mathrm{d} \zeta|\tilde{F}|^{2} \equiv \frac{m \omega^{2}}{k_{0}} N
$$

In the theory of the NLS equation, the quantity $N$ is usually termed 'number of quasi-particles'. Now we can compute the spectral energy density, which turns out to be

$$
\mathscr{E}(\lambda)=E_{1} \frac{(\lambda-\xi)^{2}+\eta^{2}}{\cosh ^{2}(\pi(\lambda-\xi) / 2 \eta)}
$$

where

$$
E_{1} \equiv \frac{4 \pi \tilde{\gamma}^{2} \mu^{2} \omega^{2} m}{V_{\mathrm{g}}^{2} k_{0}}
$$

The function $\mathscr{E}(\lambda) / E_{1}$ is depicted in figure 3 for two different values of $\alpha \equiv \eta / x$ i. It shows a maximum at $\tilde{\lambda} \equiv \lambda / \xi=1$, with width $\Delta \tilde{\lambda} \sim \alpha$, which implies that, if $\alpha \gg 1$, the peak is very narrow (see figure $3(a)$ ), making possible the appearance of interference effects. The reflection coefficient for a single impurity is now (recall that the total soliton energy is $4 \eta$ )

$$
R_{1}=\frac{E_{1} \eta^{4}}{4} \int_{-1 / \alpha}^{\infty} \mathrm{d} y \frac{\left(y^{2}+1\right)^{2}}{\cosh ^{2}(\pi y / 2)} .
$$

We will now move to the case when two impurities are present in the system, $f(x)=\delta(x)+\delta(x-D)$. The spectral density is given by

$$
\mathscr{E}(\lambda)=E_{1} \frac{\left[(\tilde{\lambda}-1)^{2}+\alpha^{2}\right]^{2}}{\cosh ^{2}[\pi(\tilde{\lambda}-1) / 2 \alpha]} \cos ^{2}\left(A\left[(\tilde{\lambda}-1)^{2}+\alpha^{2}\right]\right)
$$

where $A \equiv 2 D \mu \xi^{2} / V_{\mathrm{g}}$, and the reflection coefficient turns out to be

$$
R=2 R_{1}+\frac{E_{1}}{8 \eta} \int_{-1}^{\infty} \mathrm{d} y \frac{\left(y^{2}+\alpha^{2}\right)^{2}}{\cosh ^{2}(\pi y / 2 \alpha)} \cos \left[2 A\left(y^{2}+\alpha^{2}\right)\right] .
$$

As in the dynamical kink case, when $A \rightarrow 0$, equation (3.44) goes to the value of a single point impurity of intensity $2 \tilde{\gamma}$ and, in the opposite limit, the reflection coefficient goes to the value $2 R_{1}$, which corresponds to non-resonant, independent scattering.
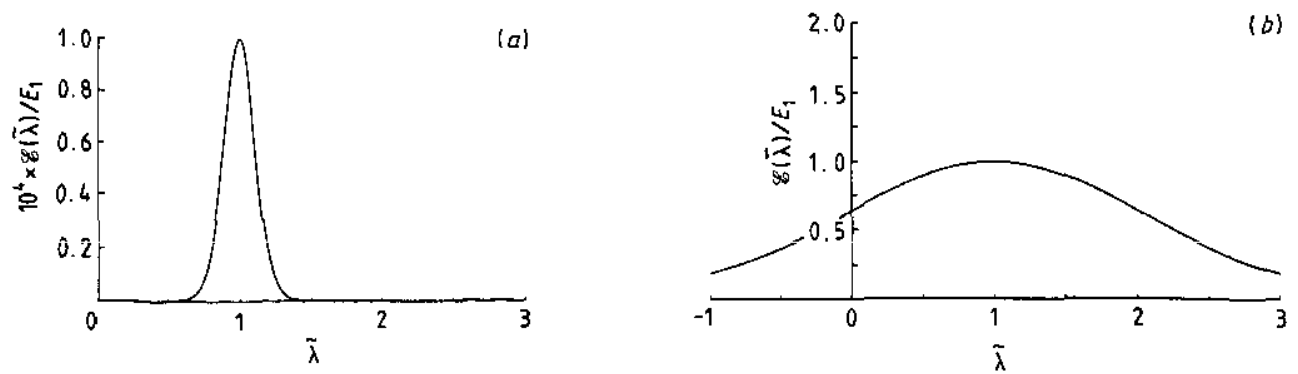

Figure 3. Spectral energy density emitted by an NLS envelope soliton in a monoatomic chain: (a) $\alpha=0.1 ;(b) \alpha=1$. 
However, a new feature appears for envelope solitons, coming from the narrow emission peak taking place when $\alpha \ll 1$ : in that limit, the reflection coefficient is very accurately given by

$$
R \approx 2 R_{1}+\frac{E_{1} \alpha}{2 \pi \eta} \mathrm{e}^{-\pi / \alpha} \cos (2 A) .
$$

It is clear from this expression that in this case resonant scattering takes place, showing itself through the oscillatory dependence of the reflection coefficient that appears in figure 4. Again, the agreement between our predictions and the numerical simulations on the same problem by Qiming et al [6] is rather good, as can be inferred from comparison of their figure 8 with our predictions.
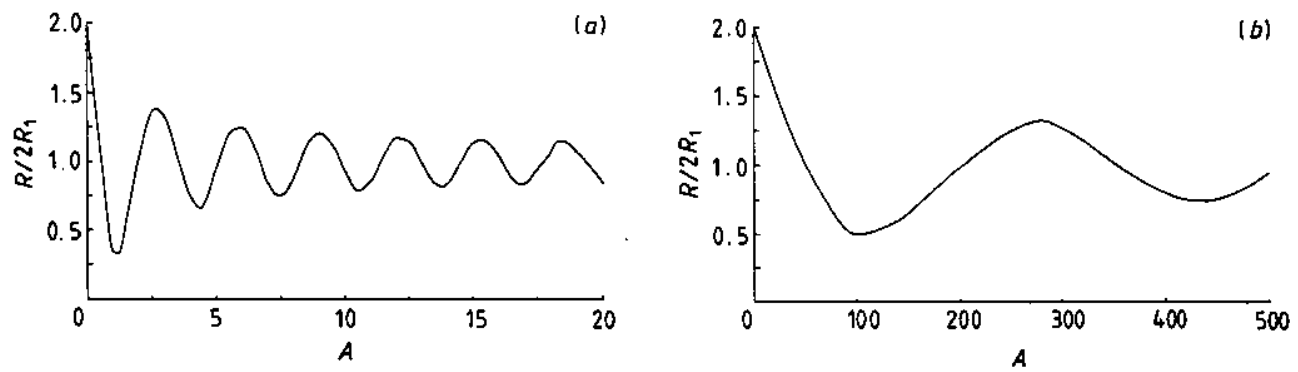

Figure 4. Ratio $R / 2 R_{1}$ for the envelope soliton against the parameter $A$ defined in equation (3.43): (a) $\alpha=0.1$; (b) $\alpha=1$.

\section{Kink-impurity interactions}

In the previous section, we have shown that envelope solitons exhibit resonance scattering phenomena upon collision with two point-like impurities, while dynamical kinks do not show such a behaviour. To complete the picture of soliton interaction with impurities, we have to deal with topological solitons. The standard examples of topological solitons are the kink solutions of the completely integrable sine-Gordon (sG) equation and the ones of the non-integrable $\phi^{4}$ system. In these equations, the kink energy has a non-zero minimum which corresponds to the static solution, so it is to be expected that the most natural effect of impurities on the kink soliton will be its pinning [14]. This pinning may be easily explained in the simple framework of the adiabatic approach, which we described in section 2.1 , considering the kink as an effective particle [14] (see also [2]). It is by no means a trivial question to ask whether topological solitons will also show the signature of their wave nature through interference effects; note that topological kinks have more particle-like characteristics than dynamical solitons or envelope solitons (these even have an internal frequency due to the carrier wave that can be expected to give rise to interference). Our main aim in this section is to answer this question.

The model we deal with is that of an inhomogeneous sG system which, in dimensionless units, is described by the equations

$$
\begin{aligned}
& u_{t t}-u_{x x}+\varepsilon(x) \sin u=0 \\
& \varepsilon_{2}(x)=1+\varepsilon[\delta(x)+\delta(x-D)] .
\end{aligned}
$$


From the physical point of view, the model (4.1), (4.2) corresponds to a long Josephson junction (LJ) with point-like inhomogeneities, $\boldsymbol{u}$ being the normalized magnetic flux [15]. Recently, junctions with such inhomogeneities, having $\varepsilon>0$ (so-called microshorts) have been constructed and studied experimentally [16-18]. The parameter $\varepsilon$ is related to the barrier thickness modulation that leads to a spatial dependence of the junction critical current. This dependence may be modelled by a delta function if the inhomogeneity length is less than the Josephson penetration length [16]. The parameter $D$ in equation (4.2) is the distance between impurities. A related problem, for finite-size inhomogeneities, has been already considered in [19]. At $\varepsilon=0$, the homogeneous sG model supports uniform motion of kinks, the expression being

$$
u_{k}(x, t)=4 \sigma \tan ^{-1} \mathrm{e}^{z} \quad z \equiv \frac{x-\zeta}{\sqrt{1-v^{2}}}
$$

where $\zeta \equiv v t+\zeta_{0}$ is the kink's coordinate, $v$ is its velocity, and $\sigma= \pm 1$ is its polarity; in the following we will assume $\sigma=+1$ without loss of generality. In LJJ theory, the kink solution (4.3) describes a magnetic flux quantum, hence termed fluxon, propagating along the junction [15].

Once more, we will apply the technique described in section 2 . Simple calculations allow us to obtain the spectral density of the kink emission upon collision with one or two impurities in the form (see details of the calculations in [2])

$$
\begin{aligned}
& \mathscr{E}(k)=4 \varepsilon_{1}(k) \cos ^{2}\left\{\frac{D}{2 v}[k v-\omega(k)]\right\} \\
& \mathscr{E}_{1}(k)=\frac{\pi \varepsilon^{2}}{8 v^{6}}\left(1-v^{2}\right)^{2} \frac{[k-\omega(k)]^{2}}{\cosh ^{2}\left[\pi \sqrt{1-v^{2}} \omega(k) / 2 v\right]}
\end{aligned}
$$

where $\omega(k) \equiv \sqrt{1+k^{2}}$, and $k$ is the wavenumber. The function (4.5) is the energy density corresponding to a kink scattered by a single (isolated) impurity. Thus, the results (4.4), (4.5) allow us to compute directly the soliton reflection coefficients

$$
R_{n}=\frac{1}{8} \sqrt{1-v^{2}} \int_{0}^{\infty} \mathrm{d} k \mathscr{C}_{n}(-k)
$$

where $n=1,2$ stands for the case with one or two impurities, respectively.

Let us analyse the structure of the kink radiation for small and large velocities. For small $v\left(v^{2} \ll 1\right)$, the spectral density $\mathscr{E}_{1}(k)$ has a single maximum at $k=0$ that is quite narrow, its width being of order $2 v / \pi \sim v$. As a consequence, the maximum will provide the main contribution to the emitted energy and give rise to a resonant dependence. It is possible to obtain the value $R_{2} / 2 R_{1}$ from equations (4.4)-(4.6) as an approximation when $v^{2} \ll 1$ as

$$
\frac{R_{2}}{2 R_{1}} \simeq 1+\frac{1}{\left(1+D^{2} / \pi^{2}\right)^{1 / 4}} \cos \left[\frac{D}{v}-\frac{1}{4} \tan ^{-1}\left(\frac{D}{\pi}\right)\right] .
$$

Notice that if $D \rightarrow 0$, we find once more the value corresponding to a single impurity with double intensity; the opposite limit yields of course $R_{2}=2 R_{1}$. On the other hand, if the speed is large, there are two maxima at $k_{1,2}= \pm k_{m}, k_{m}=2 v / \pi \sqrt{1-v^{2}} \simeq\left(1-v^{2}\right)^{-1 / 2}$. However, the function $\varepsilon_{1}(k)$ is not exponentially small in the region $|k|<k_{m}$, and hence, after averaging over all wavenumbers the oscillatory dependence disappears. 
Indeed, the asymptotics for large velocities is

$$
\frac{R_{2}}{2 R_{1}} \approx 1+3\left(\frac{d}{\sinh d}+\frac{2 \cosh d}{\sinh ^{2} d}-\frac{2 d \cosh ^{2} d}{\sinh ^{3} d}\right)
$$

where $d \approx 2 D / \sqrt{1-v^{2}}\left(D \sqrt{1-v^{2}}\right.$ must hold also in this situation). The limits $D \rightarrow 0$ or $D \rightarrow \infty$ lead to the same good results again.

Thus, we have proved that our perturbative procedure predicts resonant scattering of slow kinks. Nevertheless, another question immediately arises: recall that kinks are pinned by attractive $(\varepsilon<0)$ impurities or reflected by repulsive $(\varepsilon>0)$ impurities if their velocity is under a certain threshold. This threshold can be estimated very easily through the adiabatic approach. Substitution of $f(x)=\delta(x)$ in (2.6) yields [2]

$$
U_{\mathrm{efr}}(\zeta)=2 \varepsilon \operatorname{sech}^{2} \zeta
$$

where $\zeta$ is the kink's coordinate defined in (4.3), which in this approach is treated as a collective variable. It is clear that for $\varepsilon>0$ the potential is repulsive, and the threshold velacity below which the kink is repelled by the impurity can be defined by a simple relationship such as

$$
\frac{1}{2} m v_{t h r}^{2}=U_{\max }
$$

where $U_{\max } \approx 2 \varepsilon$. For instance, substitution of $\epsilon=0.1$ and $m=8$ into (4.10) yields $v_{t h x} \approx \sqrt{0.05} \approx 0.224$, which is not at all a small value. So, it might be possible that our perturbative theory does not have any applicability range. The only way to check if this is so or not is to perform numerical simulations of the model (4.1), and that is what we report in the remainder of this section.

The numerical procedure we use to simulate the kink scattering is the finite difference scheme of Strauss and Vázquez for the nonlinear Klein-Gordon equation [20]. This procedure has been successfully employed to study a number of different perturbed nonlinear Klein-Gordon problems and it exhibits many good properties (see [21] and references therein). Moreover, the most important property of the discrete scheme is that it exactly conserves the energy of the system in the unperturbed evolution, and it reproduces the variation of energy when the system is under the infuence of timedependent perturbations, even if they are strong $[21]$. This is relevant to the computation we intend to do, because it essentially involves precise energy calculations. Details on the scheme can be found in the literature $([20,21]$ and references therein), and hence we will not go into further detail here.

To discuss the relationship between analytical and numerical results, let us first specify the way we compute the refection coefficient. We start the simulation with a kink with a certain initial velocity, its centre sufficiently fat away from the first delta function, at $x=0$, typically 5 units ( $=$ the kink width in dimensionless units). We let it collide with the two impurities and monitor the amount of energy that is contained in three zones: at the left of the first $\delta$, i.e. at $x<0$; between both $\delta s, 0<x<D$, and at the right of the second one, $x<D$. When the kink has passed through the second impurity, and the amount of energy in the middle is negligible (less than an order magnitude smaller than the one in the left, usually two or three orders of magnitude except for the largest $D s$ ) we stop the simulation, because then the correction due to the energy that has not still travelled backwards to cross $x=0$ is very small. The reflection coefficient is finally computed straightforwardly as the ratio between the energy in the left and the initial energy of the kink, the latter being conserved as the total energy of the system. 
The numerical results for two values of the initial kink velocity are shown in figures 5 and 6, where both theoretical and numerical results are plotted; in both figures, the full lines correspond to the analytically computed dependence given by equations (4.4)-(4.6). In addition, for $v=0.4$ we performed some simulations for attractive delta functions, $\varepsilon=-0.1$, shown in full circles, about which we will comment later. As can be seen, the agreement between analytical and numerical curves is good. The main differences arise at $v=0.4$ (figure 5); in particular, the asymptotic behaviours of the analytical and numerical curves are not the same. This disagreement, that in principle should not be expected, can be explained in a natural way. Indeed, the analytical results for two impurities were obtained under the assumption that the kink does not change its velocity during the scattering (Born approximation). However, as a matter of fact, after the first scattering the kink loses some part of its kinetic energy, so that it interacts with the second impurity at a smaller velocity, say $v-\Delta v$. Hence, the ratio $R_{2} / 2 R_{1}$ does not tend to 1 when the distance between deltas, tend to infinity; rather

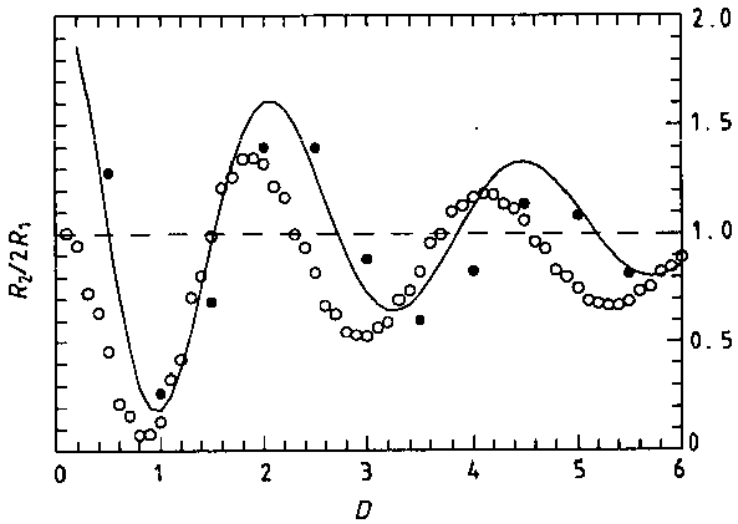

Figure 5. Dependence of the reflection coefficient on the distance between impurities for a kink with $v=0.4$ when the impurity is repulsive ( $\varepsilon=0.1$, empty circles) or attractive ( $\varepsilon=-0.1$, full circles). The ratio of the coefficient to twice the reflection coefficient for one impurity is plotted. Full line: analytical calculation taken from equations (4.4)-(4.6).

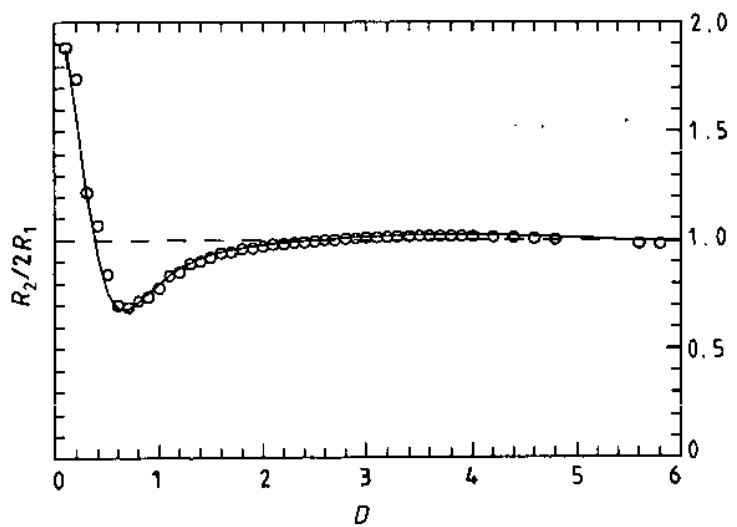

Figure 6. As in figure 5 but with $v=0.9$. Only reflection coefficients for attractive impurity are shown. 
well, it verifies

$$
\frac{R_{2}}{2 R_{1}} \rightarrow \frac{\left[E_{\mathrm{em}}^{(-)}(v)+E_{\mathrm{em}}^{(-)}(v-\Delta v)\right]}{2 E_{\mathrm{em}}^{(-)}(v)}
$$

$E_{\text {em }}^{(-)}$being the energy reflected by a single impurity. To understand the difference between $E_{\mathrm{em}}^{(-)}(v)$ and $E_{\mathrm{em}}^{(-)}(v-\Delta v)$, we have analysed the emitted energy for a single impurity versus the kink velocity. Figure 7 presents both numerical and analytical dependences. From these plots, it turns out that when the initial velocity is $v=0.4$, $E_{\mathrm{em}}^{(-)}(v)>E_{\mathrm{em}}^{(-)}(v-\Delta v)$, so that the asymptotics of $R_{2} / 2 R_{1}$ computed numerically is always smaller than the analytical predictions. This ceases to be true when $v$ becomes larger than $v_{\mathrm{cr}} \simeq 0.6$ because in that range $E_{\mathrm{em}}^{(-)}(v)$ decreases and hence $E_{\mathrm{em}}^{(-)}(v-\Delta v)>$ $E_{\mathrm{em}}^{(-)}(v)$. Thus, in figure 6, the numerical asymptotics is slightly above the line $R_{2} / 2 R_{1}=$ 1 , the difference being small due to the short time that this kink takes to cross the distance $D$ between, much shorter than in the other case.

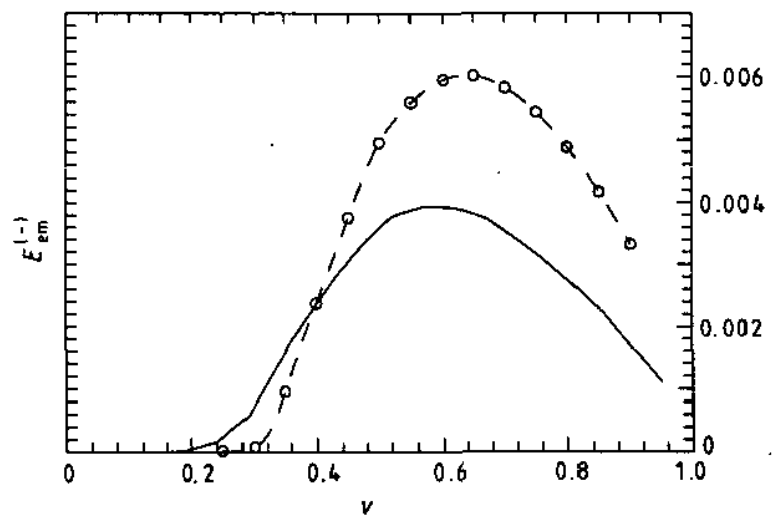

Figure 7. Energy $E_{\mathrm{em}}^{(-)}$emitted during the kink scattering by one impurity with $\varepsilon=0.1$, as a function of the kink velocity. Full line: analytical calculation. Circles: values as obtained from numerical simulations; the dashed line is a cubic spline fitting to the numerical points.

Let us briefly discuss the kink scattering by attractive inhomogeneities, $\varepsilon<0$ in (4.2). It is easy to see from equations (4.4)-(4.6) that the analytical approach based on the Born approximation give the same results, because all formulae of the perturbation theory are proportional to $\varepsilon^{2}$. Numerical simulations show some difference (see figure 5); for instance, the emitted energy calculated in the way discussed above is more than that of the repulsive situation. For example, the total emitted energy of a kink with velocity $v=0.4$ for a single impurity is $E_{\mathrm{em}}^{(-)}=2.377 \times 10^{-3}$ for $\varepsilon=0.1$, but $E_{\mathrm{em}}^{(-)}=3.427 \times 10^{-3}$ for $\varepsilon=-0.1$. This can be understood if we take into account the fact that, in the case of an attractive impurity, the linearized sG model supports the so-called impurity mode [22], whose expression is

$$
u_{\mathrm{im}}(x, t)=a \exp \left(-\frac{1}{2}|\varepsilon| x\right) \cos \omega t \quad \omega^{2}=1-\frac{1}{4} \varepsilon^{2} .
$$

Thus, due to the scattering process, the sG kink excite the impurity mode [23], which as it appears from (4.12) is localized in space, being important in the large region $\Delta x \sim|\varepsilon|^{-1} \simeq 10$ ( $\simeq$ kink length). This impurity mode does give an additional (but small!) contribution to the radiated energy as computed in the region $x<0$. Detailed analysis of the impurity mode excitation during kink scattering and also a resonant behaviour 
originated from an energy-exchange mechanism between the kink and this impurity mode may be found in the recent papers [24].

To conclude this section, we would like to point out that resonant scattering phenomena are not a property arising from the integrability of the unperturbed models, i.e. from the fact that the inhomogeneous models we are considering here are quasiintegrable (which is a necessary condition to apply our perturbative formalism). We can show this through some simulations on the $\phi^{4}$ model, described by the equations

$$
\begin{aligned}
& u_{t u}-u_{x x}+\varepsilon(x)\left(-u+u^{3}\right)=0 \\
& \varepsilon_{2}(x) \equiv 1+\varepsilon[\delta(x)+\delta(x-D)]
\end{aligned}
$$

and which, in the unperturbed case, is non-integrable. The results of our simulations, which will be reported in full detail elsewhere [25], are shown in figure 8 . The similarity of the behaviour of $\phi^{4}$ kinks to those of the $\mathrm{sG}$ system is completely evident from this plot. So, it is very likely that the main reason for the appearance of resonance scattering is the interference experienced by a dominant wavenumber present in the radiation emitted by kinks, this being the only common factor between the $\phi^{4}$ and sG models. Our perturbative technique, based on the IST method, is not valid for the $\phi^{4}$ model; however, a different approach can explain this behaviour [25], being of the same physical origin as for the sG system.

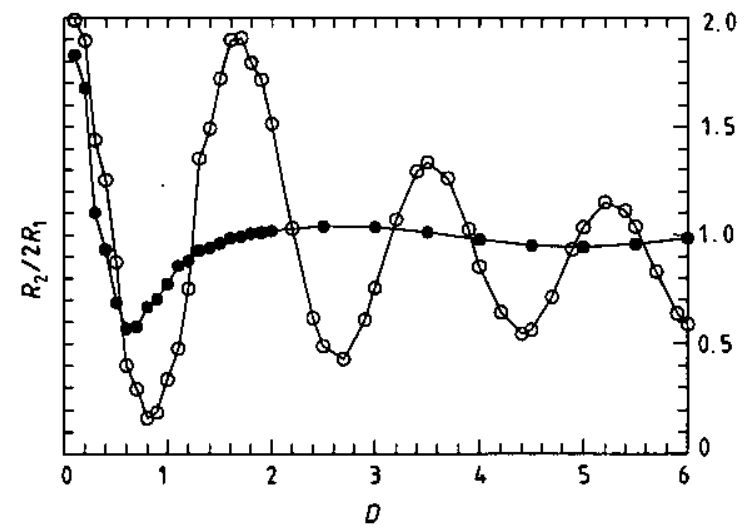

Figure 8. Dependence of the reflection coefficient on the distance between impurities for a $\phi^{4}$ kink with $v=0.4$ (empty circles) or $v=0.9$ (full circles) for a repulsive impurity $(\varepsilon=0.1)$. The full line is a cubic spline fitting in both cases.

\section{Conclusions}

We have studied the wave properties of the three main types of solitons, which play a crucial role in the scattering processes in inhomogeneous systems through interference effects. We have shown that interference is important when there is a characteristic wavelength in the spectrum of the radiation emitted by solitons upon collision with impurities. Thus, in nonlinear chains, dynamical kinks do not exhibit the peculiar oscillatory behaviour of the reflection coefficient proper to the resonance phenomena, because they emit a wide linear wave spectrum, while envelope solitons do show resonances and oscillatory reflection coefficients for small velocities, when there is a well defined peak in their emission spectrum. Our predictions are in good agreement with previous numerical simulations. The third type of solitons, topological solitons, 
present the same properties, even above the reflection-pinning threshold velocity, as we have proven numerically. We have also explained the reasons for the small discrepancies that exist between our theory and numerics. We have discarded the quasiintegrability of the models as a possible reason for this behaviour by means of simulations of the non-integrable $\phi^{4}$ model, having also resonant scattering processes. So, we must conclude that the factor responsible for resonances and different behaviours of different types of solitons is the linear wave spectrum emitted by them.

\section{Acknowledgments}

We would like to thank St Pnevmatikos and B A Malomed for useful discussions. This work has been supported in part by the Comisión Interministerial de Ciencia $y$ Tecnología (CICyT) of Spain, through project MAT90-0544. The stay of Yu S Kivshar in Madrid has been supported by the Universidad Complutense de Madrid through the Programa de Sabáticos Complutense, and he is also indebted to the Alexander von Humboldt-Stiftung for financial support through a research fellowship.

\section{References}

[1] Bishop A R, Campbell D K and Pnevmatikos St (eds) 1989 Disorder and Nonlinearity (Springer Proceedings in Physics 39) (Berlin: Springer)

Abdullaev F Kh, Bishop A R and Pnevmatikos St (eds) 1992 Nonlinearity with Disorder (Springer Proceedings in Physics) (Berlin: Springer) in press

[2] Kivshar Yu S and Malomed B A 1989 Rev. Mod. Phys. 61763

[3] Sánchez A and Vázquez L 1991 Int. J. Mod. Phys. B 52825

[4] Fogel M B, Trullinger S E, Bishop A R and Krumhansl J A 1976 Phys. Rev. Lett. 36 1411; 1977 Phys. Rev. B 151578

[5] Kivshar Yu S 1992 Nonlinearity with Disorder (Springer Proceedings in Physics) ed F Kh Abdullaev, A $R$ Bishop and St Pnevmatikos (Berlin: Springer) in press

Gredeskul S A and Kivshar Yu S 1992 Phys. Rep. 2161

[6] Qiming Li, Pnevmatikos St, Economou E N and Soukoulis C M 1988 Phys. Rev. B 373534

[7] Bergmann D J, Ben-Jacob E, Imry Y and Maki K 1983 Phys. Rev. A 273345

[8] McLaughlin D W and Scott A C 1978 Phys. Rev. A 181652

[9] Zakharov V E, Manakov S V, Novikov S P and Pitaevskil 1980 Theory of Solitons. The Inverse Scattering Transform (Moscow: Nauka) English translation by Consultants Bureau, New York, 1984

[10] Flytzanis N, Pnevmatikos St and Remoissenet M 1985 J. Phys. C: Solid State Phys. 184603

[11] Collins M A 1981 Chem. Phys. Lett. 77342

[12] Yoshida F and Sakuma T 1978 Prog. Theor. Phys. 60 338; 1982 Prog. Theor. Phys. 67 1379; 1982 Prog. Theor. Phys. 6829

[13] Kivshar Yu S, Kosevich A M and Chubykalo O A 1987 Phys. Lett. 125A 35

[14] Currie J F, Trullinger S E, Bishop A R and Krumbansl J A 1977 Phys. Rev. B 155567

[15] Barone A and Paternó G 1982 Physics and Applications of the Josephson Effect (New York: Wiley)

[16] Golubov A A, Serpuchenko I L and Ustinov A V 1988 Zh. Eksp. Teor. Fiz. 94296 (1988 Sov. Phys.-JETP 67 385); 1988 Phys. Lett. 130A 107

[17] Serpuchenko I L and Ustinov A V 1988 Solid State Commun. 68693

[18] Malomed B A and Ustinov A V 1990 Phys. Rev. B 41254

[19] Kivshar Yu S, Kosevich A M and Chubykało O A 1987 Phys. Lett. 129A 449

[20] Strauss W and Vázquez L 1978 J. Comput. Phys. 28271

[21] Sánchez A and Vázquez L 1991 Phys. Lett. 152A 184

[22] Braun O M and Kivshar Yu S 1991 Phys. Rev. B 431060

[23] Fraggis T, Pnevmatikos St and Economous E N 1989 Phys. Lett. 142A 361

[24] Kivshar Yu S, Zhang Fei and Vázquez L 1991 Phys. Rev. Lett. 671177

Zhang Fei, Kivshar Yu S and Vázquez L 1992 Phys. Rev. A 456019

[25] Kivshar Yu S and Sánchez A unpublished 